\title{
Lo relativo y lo absoluto en la concepción de Paul Natorp del método de la filosofía
}

Laura Pelegrín

Universidad Diego Portales/CONICYT

Universiteit Leiden

Instituto de Humanidades

1416 Ciudad Autónoma de Buenos Aires (Argentina)

laura.pelegrin@mail.udp.cl

Abstract: Paul Natorp argues that the problem of method is the fundamental question of philosophy. However, this representative of Neo-Kantianism proposes two apparently irreconcilable methodological requirements: a) philosophy should be a first science that is independent of any other and, b) philosophy must start from the fact of science. The aim of this paper is to clarify how these two methodological requirements are combined in Natorp's system.

Keywords: Paul Natorp, Neo-Kantianism of Marburg, method.
Resumen: Paul Natorp sostiene que el problema del método es el problema fundamental de la filosofía. Sin embargo, este representante del neokantismo propone dos prescripciones metodológicas en apariencia irreconciliables: a) la filosofía debe ser una ciencia primera que no dependa de ninguna otra y b) la filosofía debe partir del hecho de la ciencia. El objetivo de este trabajo es esclarecer cómo se conjugan estas dos exigencias metodológicas en el sistema del filósofo.

Palabras clave: Paul Natorp, neokantismo de Marburgo, método. 


\section{LAURA PELEGRÍN}

\section{INTRODUCCIÓN}

$\square$ 1 problema del método es el leitmotiv del neokantismo de Marburgo $^{1}$. Ernst Cassirer, al ser interrogado por Heidegger en el encuentro en Davos respecto al rasgo determinante de la escuela, responde que su elemento distintivo no es ninguna teoría en sí misma sino un método. El neokantismo, observa Cassirer, debe ser comprendido "funcionalmente" y no "sustancialmente". Esta tradición de pensamiento no se definiría por una teoría fija e invariable sino por un modo de poner los problemas, por un modo de filosofar ${ }^{2}$. Kant y la escuela de Marburgo es, en este sentido, el manifiesto de la escuela. Aquí Natorp señala que uno de los descubrimientos más sobresalientes de Kant ha sido el del método transcendental. Para Natorp, y para la escuela de Marburgo en general, este método se identifica con el método analítico kantiano. El método analítico que utiliza Kant en los Prolegómenos es la vía legítima de investigación de la filosofía. La filosofía debe partir del hecho de las producciones del pensamiento para luego retrotraerse a sus condiciones de posibilidad. El método resulta así el rasgo determinante de la actividad filosófica ${ }^{3}$.

No obstante, encontramos en el sistema de Natorp dos elementos característicos de su propuesta metodológica cuya integración no resulta evidente por sí misma. En su análisis de la tarea que atañe a la labor filosófica, Natorp sostiene que la filosofía es la ciencia de los primeros principios. Para Natorp, el objetivo de la filosofía es esclarecer los principios últimos del pensamiento. Estos elementos últimos serán las condiciones generales de todo pensar y determinarán así a todo pensamiento particular. La filosofía como ciencia no depende de ninguna otra y es, en este sentido, ciencia primera. Sin embargo, otro de los rasgos distintivos de la propuesta de Paul Natorp consiste en la concepción de que la filosofía debe comenzar su tarea en el progreso de la ciencia. La ciencia en su

1. H. HolzheY, Cohen und Natorp (Schwabe, Basel/Stuttgart, 1986) 50.

2. M. Heidegger, Kant und das Problem der Metaphtysik. Gesamtausgabe, Bd. 3 (Vittorio Klostermann, Frankfurt am Main, 1991) 274.

3. P. NATORP, Kant und die Marburger Schule, "Kant-Studien" 17 (1912) 193-221, 72. 
evolutivo devenir es el punto de partida de una indagación filosófica. No hay ningún factum absoluto para el pensamiento sino una continua progresión de su expresión en el saber científico. De este modo, se presenta un posible conflicto entre las prescripciones metodológica natorpianas. Por un lado, la filosofía busca principios últimos $\mathrm{y}$ fundamentales $\mathrm{y}$, por ello, no puede depender de ninguna otra ciencia. Por otro lado, la filosofía debe comenzar por un momento en apariencia extrínseco, i.e., el desarrollo de la ciencia en su infinito progreso. El problema es cómo conjugar estas dos exigencias del filósofo: a) la filosofía debe ser una ciencia primera que no dependa de ninguna otra y debe buscar principios fundamentales, b) la filosofía debe comenzar su indagación en el fieri del pensamiento científico. La ciencia es el punto de partida de la investigación filosófica; no como un hecho absoluto sino en su constante progreso en el que todo logro alcanzado es siempre relativo. Encontramos así una doble problemática. En primer lugar, de acuerdo con el primer señalamiento, la filosofía debe ser ciencia primera y en su búsqueda de los primeros principios no puede depender de ninguna otra ciencia. Sin embargo, de acuerdo con la segunda prescripción metódica, la filosofía debe comenzar por el factum del desarrollo científico. En este sentido, la filosofía pareciera depender de la ciencia en tanto ésta constituye su punto de partida. Se podría pensar que esto atenta contra la primera prescripción metódica que reclama la autonomía de la filosofía como ciencia fundamental. En segundo lugar, como observaremos, este peculiar hecho por el que debe comenzar la filosofía es un progreso. No hay en verdad ningún hecho absoluto sino siempre alcances relativos. Sin embargo, la concepción de la tarea de la filosofía implicaba que diera con principios últimos. Luego, el problema es cómo conjugar estos dos elementos. Por un lado, el carácter esencialmente autónomo de la filosofía y su pretensión de una legitimidad inmanente y, por otro, el que deba comenzar su tarea por el hecho de la ciencia en su constante devenir ${ }^{4}$.

4. La conjugación de estos dos elementos ha llamado la atención de los estudiosos del pensamiento de Natorp. Vasilis Politis, por ejemplo, observa: "This emphasis on the revisability and open-endedness of thought is central in Natorp, and it is striking that he thinks it compatible with the transcendental method (...)". 
El objetivo de este trabajo es esclarecer el vínculo entre estos dos momentos determinantes del sistema de Natorp, exhibir cómo se reúnen en su propuesta metodológica. Pretendemos mostrar cómo lo relativo y lo absoluto se conjugan en la empresa del filósofo. Procederemos en tres etapas. En primer lugar, estudiaremos la idea natorpiana de la filosofía como ciencia primera. En segundo lugar, analizaremos el argumento de Natorp de acuerdo con el cual la filosofía debe comenzar por el hecho de la ciencia. Finalmente, a modo de conclusión, exhibiremos cómo estos dos elementos de la filosofía natorpiana, en principio heterogéneos, se conjugan en la propuesta del filósofo.

\section{LA FILOSOFÍA COMO CIENCIA PRIMERA: LA LEGITIMACIÓN \\ INMANENTE Y LA BÚSQUEDA DE CONCEPTOS FUNDAMENTALES}

Para Natorp, la filosofía es aquella ciencia que tiene a cargo la tarea de desplegar los principios fundamentales del pensar. Su meta es establecer la legalidad del conocer objetivo ${ }^{5}$. Esta ciencia se constituye en este sentido determinado en la ciencia fundamental del conocer ${ }^{6}$. La tarea que debe cumplir es hallar las leyes fundamentales del pensamiento. La labor del filósofo es dar con estos principios primeros.

A lo largo de su obra, Natorp brinda las exigencias metodológicas generales para llevar a cabo esta empresa. Explicita aquellas condiciones que le permiten a la filosofía lograr este fin que se presenta como el más alto para el pensamiento. Este fin es el más elevado porque será aquel que posibilite establecer la legalidad

V. POLITIS, Anti-Realist Interpretations of Plato: Paul Natorp, "International Journal of Philosophical Studies" 9/1 (2001) 47-61, 50.

5. Natorp emparenta pensar y conocer. El desarrollo más conciso de esta cuestión se encuentra su Logik 1910. P. NATORP, Logik (Grundlegung und logischer aufbau der mathematik und mathematischen naturwissenschaft) in leitsatzen zu akademischen vorlesungen (N. G. Elwert, Marburg, 1910) \$2.

6. "Philosophie ist nach ihrem historischen Begriff die Grundwissenschaft, d. h. diejenige Wissenschaft, welche die Einheit der menschlichen Erkenntnisse durch den Nachweis des gemeinsamen letzten Fundaments, auf dem sie alle ruhen, sicherstellen soll." P. NaTORP, Philosophische Propädeutik (Allgemeine Einleitung in die Philosophie und Anfangsgründe der Logik, Ethik und Psychologie) in Leitsätzen zu akademischen Vorlesungen. (N. G. Elwert, Marburg, 1905) \$1. 
general del pensar y, a partir de aquí, fundar los restantes ámbitos del saber. Al comienzo de la Propedéutica Filosófica, Natorp establece una caracterización general de la filosofía calificándola como ciencia fundamental. Al igual que gran parte de la tradición filosófica, señala que su objetivo será encontrar aquel fundamento último que asegure la unidad de los conocimientos humanos. La filosofía, como base última de todo saber, no puede depender de ninguna otra ciencia. La filosofía debe ser autónoma. Por lo tanto, debe fundamentarse a sí misma y, a partir de allí, brindar los cimientos para las restantes ciencias ${ }^{7}$. Esta ciencia de los primeros principios es, en este sentido, ciencia de ciencias. Su objeto no es ninguno en particular sino la determinación de la totalidad del saber en general. Por ello, esta disciplina debe fundarse a sí misma para fundar luego las restantes áreas del saber ${ }^{8}$. La labor filosófica debe buscar su legitimidad de modo inmanente y ser principio determinante de las restantes ciencias. Esto es lo que Natorp denomina el "criterio formal" que debe cumplimentar para constituirse en la ciencia primera buscada. A este criterio formal de autofudamentación se le agrega un "criterio material". Esta disciplina, como centro nuclear del conocimiento, debe delimitar las regiones de los restantes objetos del saber. Sin embargo, no debe ser pretensión suya el contribuir al contenido de las ciencias particulares, sino brindar los principios últimos del pensamiento en general y, consecuentemente, la normatividad elemental en la que se despliegan los otros ámbitos del conocimiento humano. Es decir, la filosofía no determinará el contenido material de las ciencias sino que, más bien, dará los principios lógicos fundamentales de su proceder. Para lograrlo, debe fundarse a sí misma. La filosofía requiere una legitimación inmanente. De este modo,

7. Cf. P. NATORP, Philosophische Propädeutik, \$7 y P. NATORP, Über objective und subjective Begründung der Erkentnnis, "Philosophische Monatschefte" Bd XXIII Heidelberg (1887) 257-286, 257.

8. "Denn Philosophie will allerdings Wissenschaft sein, nicht aber besondere eines besonderes Gegenstandes: vielmehr eben, was allen Sonderungen der vielen Wissenschaften gegenüber der Einheit der Wissenshaft ausmacht und begründet: das ist ihre eigentümlich Frage und Ausgabe." P. NATORP, Philosophie ibr Problem und ibre Probleme: Einfuibrung in den kritischen Idealismus (Vandenhoeck \& Ruprecht, Götingen, 1911) 3 . 
la circularidad de fundamentación se introduce desde un principio como una prescripción metódica primaria porque es demandada por el fin perseguido. En tanto lo que se busca es una ciencia primera, ésta no puede depender en sus principios de ninguna otra ciencia. $\mathrm{Su}$ tarea consiste en auto-fundamentarse para brindar condiciones de legitimidad a los otros ámbitos del saber. A cada ciencia o teoría particular pertenece un campo o región de objetos determinados. Lo que se busca en un sistema filosófico es un encadenamiento coherente de leyes de modo tal que todas las leyes particulares de cada región temática se integren en un sistema total unificado. La filosofía, como ciencia fundamental, tendrá como objetivo mostrar la estructura legal interna de este sistema. Se deberá indagar en virtud de qué condiciones son conocidos los objetos en la experiencia; cuáles son los requisitos que debe cumplimentar el conocimiento de objetos para ser calificado como auténtico. Por ende, hay una circularidad virtuosa interna al método; la filosofía, como ciencia general del conocimiento, tiene que tener una legitimación inmanente. Esta exigencia surge como prescripción inicial con el fin de garantizar el estatus epistémico propio de una ciencia primera.

A partir de aquí, podemos deslindar tres rasgos fundamentales de la concepción natorpiana de la filosofía. En primer lugar, la filosofía es la ciencia de los primeros principios. Debe buscar los principios últimos del saber, sus conceptos fundamentales. Su materia de estudio no es ningún objeto en particular sino la unidad del saber en general. En segundo lugar, y como consecuencia de su objetivo, la filosofía no puede depender de ninguna otra ciencia. Debe fundarse a sí misma y fundar de este modo la unidad de los múltiples ámbitos del conocer. Este segundo requisito surge en relación al primero. En tanto los que se busca son principios fundamentales, la empresa filosófica no puede de ninguna otra ciencia. En tercer lugar, por ser la filosofía ciencia general del saber, debe brindar los principios generales de todo conocer objetivo. En este sentido es normativa respecto de todo conocer regional expresado en las ciencias particulares, aun cuando solo determine los principios generales y no las leyes particulares de cada ciencia determinada. 


\section{EL FACTUM DE LA CIENCIA COMO PUNTO DE PARTIDA}

Sin embargo, frente a esta idea de circularidad en la fundamentación Natorp también reclama que la filosofía tenga una relación directa con el hecho que se debe elucidar. La filosofía debe tener una firme correlación con los facta históricamente determinables. Natorp reniega de los estudios puramente especulativos y propone el establecimiento de un método reductivo de análisis. La filosofía debe encontrar el elemento primero para iniciar su tarea. La circularidad inmanente no será por ello puramente especulativa. El pensamiento deberá comenzar por un hecho determinado para establecer su legalidad propia. De aquí se sigue otra de las exigencias del método crítico: lo que se debe es obtener el fundamento de derecho o razón de ser de cada uno de los eventos bajo estudio. De acuerdo con el filósofo de Marburgo, todo "lo que es" resulta de una objetivación de un acto del intelecto. Para Natorp, todo ser es ser para el pensar. No hay ser fuera del pensamiento9. Por ello, la legalidad de dicha objetivación representa no sólo su condición de inteligibilidad sino, asimismo, su razón de ser. Las condiciones de legitimidad del conocer serán tanto epistémicas como ontológicas ${ }^{10}$. Por ello, Natorp

9. Como observa Arnaud Dewalque el rechazo natorpiano a toda idea de multiplicidad dada se asienta en última instancia en dos argumentos. El primero, se focaliza en el rechazo al empirismo ingenuo; el segundo, en el rechazo al absolutismo del idealismo especulativo A. Dewalque, La critique néokantienne de Kant et l'instauration d'une théorie conceptualiste de la perception, "Dialogue" 49 (2010) 413-433, 422. No obstante, Dewalque omite destacar el argumento positivo de Natorp. Natorp prueba que el concepto de múltiple se desprende del acto de pensamiento, y no hay nada que lleve a inferir un elemento dado: P. NATORP, Die logischen Grundlagen der exakten Wissenschaften (B. G. Teubner, Leipzig-Berlin, 1910) 8ss.

10. Para Natorp, la construcción de una lógica del conocimiento es, al mismo tiempo, una ontología general. En este sentido, llama la atención que Vasilis Politiz contraponga lo lógico a lo ontológico. Particularmente, considerando que en otro de sus comentarios, este mismo comentador afirma que Natorp construye una ontología general a partir de una epistemología transcendental Cf. V. POLITIS, Anti-Realist Interpretations cit., 52 y V. PoliTIS, An Introduction to Paul Natorp's Plato's Theory of Ideas en P. Politis, Plato's Theory of ideas. An Introduction to Idealism (Trad. V. Politis, John Connolly, 2004), 38. Así también, si comprendemos a la metafísica como ontología, no habrá que esperar a los desarrollos tardíos de Natorp para encontrar el desarrollo de una metafísica, como considera Franz Josef Wetz. Cf. F. WeTz, Die Überwindung des Marburger Neukantianismus in der 
puede afirmar que el método trascendental representa el camino de ascenso desde el hecho de experiencia a su condición (ratio) última de $\operatorname{ser}^{11}$. Estas condiciones trascendentales no son trascendentes a la experiencia sino que, en cuanto condiciones de la misma, le son inmanentes. Esto representa que no hay una ley externa que estructure al hecho. Por el contrario, la formulación teórica sólo explicita su legalidad intrínseca. La filosofía busca la ley de estructuración objetiva en la proyección de la misma, en la creación de la vida cultural humana que se encuentra siempre en proceso y no es nunca una obra concluida.

El método acorde a estas exigencias será el crítico; éste indagará las condiciones generales que debe satisfacer todo conocimiento de objetos que se postule como verdadero. Sin embargo, como se ha señalado, las condiciones de legitimidad no son dadas ellas mismas sino que se requiere de un acto de reflexión a partir de la objetivación de la ley en el hecho. La reflexión consiste en ir desde lo objetivado a sus condiciones de objetivación, al acto que le dio origen. El método parte del hecho y se retrotrae a sus condiciones de posibilidad. Por ello, lo primero a determinar es cuál es propiamente aquel hecho a partir del cual, explicitando su normatividad propia, se pueda dar con las leyes generales de todo conocer objetivo.

\section{a) La discusión con la fenomenología y el psicologismo}

Se debe mostrar cuál es el factum privilegiado a partir del cual comenzar la indagación. En discusión con la escuela fenomenológica, Natorp argumenta que este hecho es el de la ciencia y no el de la experiencia natural.

Spätphilosophie Natorps, "Zeitschrift für philosophische Forschung" 47/1 (1993) 75-92, 76. Por el contrario, como observa Vuillemin, la metafísica natorpiana ya se encuentra desplegada en sus escritos tempranos. J. VuILLEMIN, L'béritage kantien et la révolution copernicienne. Fichte-Coben-Heidegger (Press Universitaires de France, Paris, 1954) 135ss.

11. "Der schöpferische Grund aber aller solchen Tat der Objektgestaltung ist das Gesetz; zuletzt jenes Urgesetz, das man noch immer verständlich genug als das des Logos, der Ratio, der Vernunft bezeichnet." P. NATORP, Die logischen Grundlagen cit., 197. 
El filósofo reconoce las peculiaridades que la fenomenología otorgaba a la experiencia ordinaria; el conocimiento natural es el que está ligado inmediatamente a los objetos. La inmediatez es, de hecho, una de las condiciones fundamentales de las que se sirve la fenomenología para tomar el conocimiento espontáneo como factum privilegiado. Natorp, reconociendo la inmediatez de esa clase de relación cognoscitiva, mostrará por qué esto no es condición suficiente para otorgarle el privilegio que le daba la fenomenología. El problema de este tipo de conocimiento es su unión a la relación perceptiva presente. El conocimiento espontáneo, que parte de la percepción, "se da siempre en la presencia de objetos", o, dicho fenomenológicamente, "tiene al objeto en carne y hueso" ${ }^{12}$. El filósofo reconoce ciertas categorías fundamentales del conocimiento espontáneo pasibles de ser expresadas en una unidad sistemática. No obstante, estas peculiaridades del conocimiento natural serán justamente para él las que impidan caracterizar este hecho más inmediato como aquel factum a partir del cual, de la reducción a su legalidad propia, obtener la legalidad del restante ámbito de objetos. La experiencia natural está anclada en la percepción inmediata, mientras que la prosecución del sistema explicativo exige volverse sobre estas percepciones para constituirlas como representaciones. Para Natorp, no hay posibilidad de un acceso inmediato a las vivencias. Aun el camino para un análisis psicológico, que pretenda alejarse del ámbito de lo meramente empírico, puede sólo volverse regresivamente desde la objetivación del espíritu humano a su origen. Pero, de acuerdo con Natorp, la metodología que parte de la vivencia ordinaria deviene absolutista, ya que detiene el flujo de objetivación del vivir en elementos conceptuales estancos que no pueden correr en paralelo al progreso del espíritu. Por el contrario, el método crítico no se enfrenta ante el peligro del absolutismo. Frente a este modo regresivo absolutista, Natorp sostiene que el método regre-

12. "No hay teoría concebible capaz de hacernos errar en punto al principio de todos los principios: que toda intuición en que se da algo originariamente es un fundamento de derecho del conocimiento." E. HussERL, Ideas relativas a una fenomenología pura y a una filosofía fenomenológica. Trad. José Gaos (FCE, México, 1997) $\$ 24$. 
sivo trascendental se despliega en concomitancia con el desarrollo del pensar. La reducción del dato a la ley se da a partir del hecho del desarrollo del espíritu (objetivado en su más alto grado en la ciencia de la naturaleza) y no a partir de ningún factum absoluto.

Esta disputa acerca de cuál es el hecho privilegiado partir del cual debe comenzar la indagación filosófica se inserta un contexto de debate más amplio. El problema central es si en la fundamentación del conocimiento el factor determinante es el subjetivo o el objetivo. El lado subjetivo representa al sujeto del conocimiento, es la actividad o experiencia del sujeto que conoce. Un estudio subjetivo del conocimiento indagará la experiencia fáctica del sujeto cognoscente $^{13}$. El lado objetivo representa lo conocido, es decir, el contenido del conocimiento. Natorp concede algunos argumentos que servirían para fundamentar una posición subjetivista ${ }^{14}$. Todo conocimiento es la relación de un sujeto que conoce con algo conocido. El conocimiento puede ser descrito como una vivencia psíquica de la conciencia subjetiva. Luego, una caracterización del conocimiento en general podría estar basada en el estudio de estas vivencias de la conciencia. Ésta, como fundadora de la legalidad del conocer, detentaría las leyes generales de la validez objetiva del conocimiento. El Profesor de Marburgo explica las razones por las cuales la postura subjetivista resulta insostenible. Aunque se conceda que para todo " $\mathrm{x}$ " conocido se requiere un acto de conocer, el problema del fundamento de la objetividad del conocimiento en general sólo puede

13. La finitud racional que realiza la actividad del conocer es sólo una "abreviatura" del espacio lógico (subjetividad absoluta) en el que se inserta. Natorp afirma: "Unser wirkliches Denken begnügt sich sozusagen mit Abbreviaturen des Denkens..." P. NATORP, Quantität und Qualität im Begriff, Urteil und gegenständlicher Erkentnnis, "Philosophische Monatschefte" Bd XXVII Heidelberg (1891) 1-32; 129-160, 16.

14. Éric Dufour señala al respecto: "Natorp valora la dimensión subjetiva de todo conocimiento (...) si podemos intentar fundar las leyes que rigen el conocimiento científico a partir del 'hecho de la ciencia' (fundación objetiva), se puede, igualmente, a partir del mismo punto de partida, remontarse hasta los proceso subjetivos que son la fuente (fundación subjetiva)". El comentador considera este punto como uno de separación de Natorp del sistema de Cohen. E. Dufour, Le statut du singulier: Kant et le néokantisme de l'École de Marbourg, "Kant-Studien" 93 (2002) 324-350, 332 . 
responderse a partir del concepto mismo de conocimiento, que se opone al objeto en cuanto éste representa la incógnita a conocer. El problema de la subjetividad fáctica que tiene a su objeto como contenido psíquico no constituye un factor relevante. El punto de vista de la subjetividad resulta inaceptable en tanto subordinaría lo que debería ser la ciencia primera a los principios de una ciencia particular: la psicología. La verdad objetiva de los principios del conocimiento en general no puede estar fundada en la vivencia subjetiva del sujeto cognoscente dado que si se hace depender la lógica de la psicología se suprime la pretensión misma de encontrar la fundamentación legal del conocimiento en general. Por ello, "la lógica no tiene nada que decir respecto del pensamiento como hecho o como proceso psicológico" porque lo que se debe encontrar son los conceptos y principios fundamentales que le den a esta ciencia primera una validez autónoma. En este sentido, la lógica es la contracara del conocimiento de la psicología ${ }^{15}$. Esta última versa sobre lo empírico del conocimiento subjetivo mientras que la primera busca los principios de validez general del conocimiento objetivo. De aquí que se defienda "la fundamentación autónoma y puramente objetiva de la verdad" ${ }^{16}$. El camino inverso haría perder el sentido mismo del concepto de verdad ya que lo que se comprende por validez objetiva es aquella que no depende fundamentalmente de la subjetividad.

Luego, la posibilidad de determinar la validez objetiva del saber depende en última instancia de la posibilidad del establecimiento de una objetividad en algún punto independiente de la subjetividad particular. Natorp identifica el tipo de independencia posible y requerida. Hay dos posibles sentidos de tal independencia. Una alternativa es que los objetos sean completamente exteriores y ajenos a la conciencia que conoce. Pero esta respuesta anularía el mismo concepto de objeto establecido puesto que, para Natorp, ser objeto es ser un término de un acto del conocimiento. Pensar es espontaneidad. La espontaneidad del pensamiento consiste en la capacidad de establecer relaciones entre elementos heterogéneos.

15. P. NATORP, Logik cit., 99.

16. P. NatorP, Über objective und subjective Begründung cit., 148. 
Los términos que exige el concepto de relación no son nada fuera de ella. Los términos no preceden a la relación sino que son establecidos por ésta como requerimientos suyos ${ }^{17}$. El objeto, como término de la relación que representa el conocimiento, no es nada fuera de esta relación. Pero el objeto se pone frente al conocimiento y, sin embargo, es fundado por éste. A partir de aquí surge el problema de cómo el objeto puede ser independiente del acto de conocimiento y, al mismo tiempo, estar fundado por el mismo. La respuesta natorpiana es que esta independencia es una abstracción. El objeto es puesto por el pensamiento pero como un problema que debe ser resuelto. En este sentido, el objeto también es dado al pensar como aquello que debe ser conocido. El pensamiento constituye al objeto como problema que debe resolverse. El objeto nunca será plenamente independiente del pensar sino que esta independencia es una abstracción. Para Natorp, la posibilidad de esta abstracción es confirmada indefectiblemente por el hecho mismo de la ciencia que efectivamente la realiza. La lógica de esta dinámica es expresada en la ciencia.

\section{b) El becho de la ciencia}

Para Natorp, la ciencia expresa la realización última del pensamiento. El procedimiento del saber científico permite, por medio de la postulación de hipótesis, anticipar el punto de vista y efectuar, de este modo, una primera limitación de su objeto. La tarea del conocimiento es considerada así como una tarea análoga a la resolución de una ecuación en la que el objeto es la "x" buscada. Esta incógnita no es completamente desconocida sino que ella misma es identificada qua incógnita. La tarea es lo "dado" para el pensamiento. "Ser dado" significa "ser una tarea a resolver"18. En el acto

17. "Dem Inhalte nach aber ist Denken: Setzen von Beziehung, nichts anderes. Beziehung fordert Termini; aber auch nicht diese gehen der Beziehung voran, sondern die Beziehung setzt auch erst die Termini". P. NATORP, Die logischen Grundlagen cit., 99.

18. "Ein Gegebensein darf nichts mehr bedeuten wollen als den Charakter der noch zu lösenden Aufgabe - Aufgabe eben des Ursprungsnachweises aus dem 
del conocer, algo concluso y definitivo constituye un contrasentido, puesto que invalida el concepto mismo de conocimiento como tarea. En este sentido, no hay nada absoluto para el pensamiento. De este modo, se establece una peculiar relación entre ser y pensar en la cual ser en general es ser algo para el pensamiento. La idea del conocimiento como tarea se refleja en la estructura fundamental que tiene la misma función del indagar. Una pregunta, observa Natorp, tiene tres dimensiones. Por un lado, tiene un tono prospectivo en un "no saber". Lo que no se sabe es aquello por lo que se indaga. La meta de la pregunta es dotar de contenido lo que aparece en primera instancia como el "no" del saber, su momento de indeterminación. Pero, la pregunta supone también un saber. Esta es la condición que posibilita todo preguntar por algo en general. Aquello sobre lo que se pregunta es lo desconocido, es una $\mathrm{x}$ a determinar. No obstante debe haber elementos que me permitan, al menos, la comprensión de aquello por lo que se pregunta. Sin este saber supuesto "faltarían las presuposiciones necesarias para la pregunta misma" ${ }^{19}$. Este es el momento retrospectivo. Hacia adelante se presenta lo aún indeterminado, aquello por lo que pregunta la pregunta. Hacia atrás están aquellas condiciones que permiten una identificación primaria sobre el objeto interrogado. En el medio, finalmente, está el saber del no saber, que es donde se conjugan los dos momentos anteriores. En este sentido hay una "anticipación del saber" reflejado por estos tres momentos. El conocimiento se representa como una ecuación. Esta es la estructura básica del pensar. El pensamiento es el que tiene la tarea de la determinación de su objeto en un avance progresivo. El pensar proporciona la ley mientras que la determinación particular de la experiencia representa la posibilidad de verificación de la misma pensada en primera instancia como norma general del pensamiento. La particularización en la experiencia da la realización

Einheitsgrunde der Erkenntnis". P. NATORP, Kant und die Marburger Schule cit., 200. En este sentido, como observa Maximo Ferrari, Natorp abandona toda concepción realista del concepto de objeto. El objeto es la tarea de construcción del pensamiento. Cf. M. FerRarI, Introduzione a il Neocriticismo (Editori Laterza, Roma, 1997) 69.

19. P. NATORP, Die logischen Grundlagen cit., 33. 
plena de la ley. La correlación con los facta se da a través de la anticipación en hipótesis. Estas anticipaciones son realizadas en investigaciones científicas. Ésta es la forma en que el idealismo se vincula con la experiencia. De este modo, por un lado, Natorp ratifica el segundo de los principios metódicos postulados (correlación con los hechos), mientras que, por otro, se opone al idealismo absoluto. Esta oposición se da en el reconocimiento al interior del sistema de lo irracional. Hay dos tipos de "irracionalidad" pasibles de ser aceptados. El tipo de irracionalidad que será descartada es la que surge bajo la noción de una entidad o ámbito exterior al pensamiento, lo irracional como lo completamente indeterminando, como lo otro absoluto respecto de lo racional. Lo que se niega es lo irracional "dado" pero incognoscible. Esto constituye un contrasentido dado que, como se señaló, ser un objeto es ser un dato potencialmente determinable para el entendimiento. Lo que el sistema de Natorp acepta es el "resto" en relación a lo determinado en el pensar. Para el idealismo trascendental, señala el filósofo, "no hay absoluto alguno en la experiencia" ${ }^{20}$. Lo que propiamente hay es un camino continuo de sucesivas determinaciones, la indeterminación de lo que puede ser determinado en los sucesivos avances del pensamiento. En la necesaria incompletitud del pensar se da el reconocimiento de un irracional relativo como un elemento estructural del sistema. Frente a lo irracional ajeno al pensar surge un irracional propuesto por el mismo pensamiento. El hecho de la ciencia no es el de un saber absoluto firmemente asentado sino el avance continuo que se expresa primeramente a través de hipótesis. El conocimiento de las ciencias está en un proceso infinito de limitación de lo ilimitado y no debe aceptarse un comienzo absoluto. Los comienzos se delimitan a través de las proyecciones en hipótesis. Por lo tanto, no hay verdaderamente un factum del saber en sentido estricto, en el sentido de un conocimiento seguro previamente delimitado. Por el contrario, cada

20. "Der dem Idealismus sich entgegenstemmende Empirismus enthüllt sich immer wieder als falscher Absolutismus, während der transzendentale Idealismus gerade den echten Empirismus bedeutet, der ein_Absolutes in der Erfahrung schlechterdings nicht anerkennt." P. NATORP, Kant und die Marburger Schule cit., 206. 
conocimiento se desarrolla a partir del "resto" del conocimiento anterior en una profundización del problema ${ }^{21}$. Sin embargo, no se trata de partir de cualquier hipótesis o presuposición. El punto de partida es la proyección misma de lo que se debe determinar realizada por el carácter aporético del conocimiento anterior; es decir, sobre la " $\mathrm{x}$ " como lo desconocido del objeto del conocimiento, el objeto no determinado sino por determinar de la experiencia en un proceso infinito ${ }^{22}$. Por esta vía, se conduce a ulteriores desarrollos a partir de cada comienzo relativo. A partir de lo irracional del saber anterior (en el sentido que Natorp le da al término) se avanza hacia perspectivas más determinadas y fundamentales. No a una respuesta final de la pregunta sino, dirá Natorp, hacia una ilimitada profundización de la misma. Éste es el significado del señalamiento según el cual el factum de la ciencia no es una posición única sino la totalidad del proceso en el avance del conocimiento.

Natorp reconoce en esto un acercamiento al sistema hegeliano. La diferencia con Hegel sería que éste consideró que la ley del pensar podía ser llevada a una absoluta inteligibilidad, a una realización última ${ }^{23}$. El sistema filosófico natorpiano sostiene, por el contrario, que el pensamiento prosigue su tarea infinitamente. La " $x$ " como incógnita es siempre pasible de una ampliación de su contenido. El error de Hegel fue pretender la racionalidad del todo

21. "So kann von keinem 'Factum' mehr im Sinne fertigen Wissens die Rede sein; jede Erkenntnis vielmehr, die ein Lücke des bisheringen Wissens schliesst, wird neue, größere Probleme hervortreiben." P. NATORP, Die logischen Grundlagen cit., 14.

22. "Nicht un beliebige, um irgend willkürliche Voraussetzungen kann es sich handeln, sondern nur un genau die Voraussetzungen, welche, erforderlich sind, un das X —den nicht bestimmten, aber zu bestimmenden Gegenstand- der Erfahrung in einem unendlichen Prozess, also nie in schlechthin abschliebbebder Weise, Schritt un Schritt zur Bestimmung zu bringen.” P. NaTORP, Die logischen Grundlagen cit., 15.

23. Seguimos la reconstrucción de Natorp. De acuerdo con el pensador de Marburgo, ésta sería la diferencia central entre su sistema y el de Hegel. No obstante, como reconocen varios especialistas, el problema es más complejo. Particularmente, considerando que, como marca Holzhey, Natorp concede el acercamiento al sistema de Hegel en puntos cardinales; por ejemplo, en la búsqueda de una superación del dualismo de intuición y concepto y entre materia y forma. H. HOLZHEY, Coben und Natorp cit., 65. 
de manera absoluta. Para la escuela de Marburgo también "todo es pensamiento y el pensamiento es del todo"24; sin embargo, el proceso creativo del espíritu es infinito y la meta es sólo una idea regulativa. El anti-absolutismo sería el elemento distintivo de la escuela neokantiana que la aleja definitivamente de Hegel. Esta es una diferencia esencial, puesto que el absolutismo hegeliano lleva a la parálisis del pensamiento y lo irracional no es sólo aceptado por la filosofía del marburgués sino un elemento estructural inherente a su sistema. La ininteligibilidad, la irracionalidad como negatividad relativa, es el "motor" del proceso. El absolutismo, sin embargo, no es para Natorp algo propio de los sistemas explicativos especulativos, se predica de todos aquellos sistemas que ven en principios últimos inamovibles las leyes del conocer. El método genético de Natorp se opone al absolutismo dogmático. En el sistema de Natorp el conocimiento es siempre progreso, no hay detenimiento ni retorno ${ }^{25}$.

\section{LO RELATIVO Y LO ABSOLUTO}

De acuerdo con el primer precepto metodológico, la filosofía no puede depender de ninguna otra ciencia. Sin embargo, Natorp considera que la filosofía debe comenzar por el hecho de la ciencia en su infinito desarrollo. Luego, surge el interrogante de cómo estas dos prescripciones metodológicas se vinculan entre sí. En base a lo expuesto hasta aquí, lidiaremos con esta problemática.

Para Natorp, como mencionamos, no hay ser por fuera del pensar. Ser es ser para el pensamiento. Todo lo que es debe ser tomado como una construcción del pensar. Un ser que no pueda

24. "Hegel aber lässt stets das Irrationale in das Rationale restlos auflösbar erscheinen, und so wird ihm Alles Denken, Denken Alles. Auch uns ist Alles Denken, Denken Alles, aber in ganz anderem Sinne”. P. NATORP, Kant und die Marburger Schule cit., 212.

25. "Erkenntnis aber ist Fortschritt nicht Stillstand oder gar Rückgang." P. NATORP, Die logischen Grundlagen cit., 11. Es decir, como señala el comentador Morris Cohen: "finalmente, lo genético se opone a lo óntico, el fieri como opuesto al factum”. M. CoHEN, Reviewed work: Die Logischen Grundlagen der Exakten Wissenschaften by Paul Natorp, "The Journal of Philosophy, Psychology and Scientific Methods" 8/25 (1911) 693-697, 694. 
ser pensado representa un contrasentido. No hay nada dado sin que haya sido puesto por el intelecto. La tarea del pensamiento consiste en relacionar. Su función es reunir el múltiple en una unidad ${ }^{26}$. El establecimiento de relaciones es su función fundamental. El pensamiento pone al mismo tiempo el múltiple y la unidad, las partes y el todo que las une. El pensamiento es "posición de relaciones" y los términos de la relación no son nada fuera de ella. El pensamiento tiene un carácter tético. Su correlato es la resolución de un problema como tarea. El pensamiento se expresa en los hechos que propone. El hecho es la objetivación del pensar. La ciencia es el lugar dónde se hace patente la operativa del pensamiento. El pensamiento se explicita paradigmáticamente en la ciencia. La ciencia es el lugar donde el pensamiento se realiza. Por ello, la filosofía deber tomar este punto de partida. El comienzo es la ciencia porque es el lugar donde la estructura funcional del pensamiento se hace asequible. De este modo, comenzar por un hecho a posteriori no representa un contrasentido respecto de la búsqueda de la filosofía de principios primeros y de autonomía.

Así, en primer lugar, la filosofía debe comenzar en el hecho de la ciencia como punto de partida porque la ciencia es el lugar de proyección más patente de la operativa relacional del pensar. Se trasluce allí su esencia más propia. En la ciencia se ve la operativa funcional del pensamiento. Luego, el hecho de que se deba comenzar por el factum de la ciencia no atenta contra el carácter de la filosofía como ciencia primera en tanto es en el desarrollo del pensamiento científico donde las categorías fundamentales del pensar se ponen de manifiesto. Así, es posible construir una ontología general a partir del hecho de la ciencia ${ }^{27}$. El pensamiento se vuelve objetivo en la

26. "Penser, c'est saisir le multiple dans l'unité", P. NATORP, Nombre, temps et espace dans leurs rapports avec les fonctions primitives de la pensée (Biblioteque du Congres international de la Philosophie, Colin, Paris, 1900) 344.

27. Como señala Vasilis Politis, es por medio de esta tesis que se establece el compromiso del filósofo de Marburgo con la ontología. A través del carácter relacional del pensar se funda una ontología mediada por la epistemología trascendental. Para Politis, el elemento focal aquí no es meramente epistémico, sobre cómo buscamos la verdad en los objetos sino, antes que nada, metafísico: los objetos son lo que son sólo en virtud de la relación con el pensamiento. Esto es lo que 


\section{LAURA PELEGRÍN}

ciencia y, por ello, la ciencia constituye su comienzo. Esto no entra en contradicción con su carácter de ciencia primera, pues la filosofía buscará en la ciencia la legalidad del movimiento del conocer, las diversas explicitaciones del pensamiento. En otros términos, el comienzo a posteriori no coarta la búsqueda de principios a priori puesto que estos principios originarios se plasman en lo a posteriori. El pensamiento se refleja en lo pensado y lo pensado es el conocimiento científico. La lógica trascendental, como nombre del método de la filosofía, es la exposición del modo de explicitación del pensamiento en la ciencia ${ }^{28}$.

En segundo lugar, la noción misma de pensamiento conlleva la noción de movilidad. La detención es el fin de todo pensar. Como expusimos anteriormente, esto lo expresa Natorp por analogía con una ecuación que debe ser resuelta. Lo que se pide no es una resolución última del saber sino un avance progresivo en la determinación de los objetos de conocimiento. De este modo, aun cuando los resultados sean siempre parciales y, luego, relativos, el carácter procesual del pensar es su rasgo determinante. Es decir, la esencia del pensar consiste en este progresar y, de este modo, la relatividad de su resultados no va en desmedro del carácter absoluto de su estar siempre en movimiento. El carácter determinante es la movilidad del pensamiento. Este es su rasgo específico. Por ello, lo relativo de los resultados es el acompañamiento del carácter progresivo del pensamiento.

legitima el frecuente pasaje de Natorp de la naturaleza del pensamiento a la naturaleza de lo real. Cf. V. PoLITIS, An Introduction cit., 38. No obstante, la definición esencial del pensamiento como establecimiento de relaciones es para algunos comentadores uno de los presupuestos fundacionales del sistema no debidamente justificados por Natorp. Según Mario Gonzáles Porta, "las dificultades giran en última instancia en torno a la asunción del carácter primitivo e indefinible de la relación, con la cual parece presuponerse justamente lo que se tata de probar". M. A. González PorTA, La teoría del número en Natorp y Cassirer, en J. ARANA (ed.), La ciencia de los filósofos. Número monográfico: "Thémata: Revista de Filosofía" 17 (1996) 199-222, 209.

28. En este sentido nos oponemos a las consideraciones de Antonio Caso, para quien el error de la escuela de Marburgo habría sido el confundir la ontología con la gnoseología. J. LunA HeRnáNDEZ, Una polémica en torno al neokantismo, "Historia Mexicana” 19/3 (1970) 397-417. 


\section{CONCLUSIÓN}

En primer lugar, analizamos lo que entiende Natorp por filosofía, cuál sería de acuerdo con el filósofo su objeto y su método. Observamos que Natorp otorga a la filosofía el carácter de ciencia primera. Esta ciencia no dependería en sus principios de ninguna otra y demanda, por tanto, una legitimación inmanente. La filosofía es ciencia de los primeros principios y debe, luego, ser independiente de toda rama particular del saber. En segundo lugar, estudiamos el modo en el que Natorp argumenta que la filosofía debe proceder a partir del factum de la ciencia. La defensa de Natorp de la ciencia como punto de partida del filosofar se despliega en relación a las críticas al psicologismo husserliano y al absolutismo hegeliano. La filosofía no puede partir de ningún hecho psicológico subjetivo, como el de representación, ni de una pura especulación completamente independiente de los hechos. Es decir, la filosófica busca las condiciones de todo pensar objetivo. Su indagación no puede depender de un punto de partida que dependa de la subjetividad. Pero, la filosofía también debe correr en paralelo al desarrollo del pensamiento en sus concreciones. Luego, el punto de partida tampoco puede ser puramente especulativo. Por el contrario, el comienzo para el pensamiento es su creación más propia, i.e. el fieri de la ciencia. Así, finalmente, analizamos el modo en el que se conjuga el establecimiento de una ciencia primera general a partir del fieri del pensamiento científico efectivo.

En suma, la reflexión filosófica comienza por el hecho de la ciencia porque es en la ciencia donde se explicitan las categorías fundamentales del pensamiento. De este modo, su búsqueda de principios últimos puede llevarse a cabo aun cuando parte de un hecho en apariencia extrínseco. Este hecho es, en realidad, solo en apariencia extrínseco porque la filosofía busca la legalidad general del pensar y esta legalidad se explicita en la ciencia. Por ello, éste es su punto de partida. En segundo lugar, que este comienzo de la reflexión se dé a partir de un hecho que está un constante devenir tampoco atenta contra la búsqueda de principios primeros. El comienzo es la ciencia en su devenir porque es en este devenir que se explicitan los princi- 
pios fundamentales del pensamiento. Lo relativo de los resultados es consecuente con lo absoluto del movimiento. Así, la filosofía prescribe las leyes que rigen este devenir aun cuando los resultados que el conocimiento vaya alcanzando sean siempre relativos y dependan de la ciencia de la naturaleza. De este modo, se conjuga en armonía lo absoluto y lo relativo en la propuesta filosofía de Natorp en cuanto al método que debe seguir la filosofía.

En la contemporaneidad, varios pensadores han tomado el método de Natorp y lo han aplicado para construir principios filosóficos a partir de los nuevos desafíos que propone la ciencia. Hernán Pringe, Marco Giovanelli y Mario Ariel González Porta son algunos de los pensadores que han emprendido la tarea de reflexionar sobre la relación entre la propuesta de Natorp y problemas científicos especiales. En su Critique of the Quantum Power of Judgment. A transcendental Foundation of Quantum Objectivity, Pringe sigue el método trascendental natorpiano para dar una fundamentación epistemológica a la física cuántica; particularmente, estudia el estatus epistemológico de los objetos cuánticos a través de la aplicación de este método $^{29}$. Giovanelli centra su estudio en el problema de la relación entre lo intensivo y lo inextensivo, explicando cómo el filósofo de Marburgo se posiciona en un debate propio de la época ${ }^{30}$. González Porta analiza la teoría de Natorp del número y estudia la contribución del filósofo al estructuralismo matemático ${ }^{31}$. De este modo, estos pensadores han mostrado el valor de la propuesta natorpiana para los problemas que presenta la ciencia en la contemporaneidad. Algunos, como en el caso de Pringe, aplicando la metodología natorpiana a sus propias reflexiones filosóficas; otros, como Giovanelli

29. Esta es una tesis de lectura que puede ser discutida; particularmente considerando que Pringe sostiene que el método de su investigación es puramente sintético (i.e., progresivo) y no analítico (i.e., regresivo). H. PRINGE, Critique of the Quantum Power of Judgment. A transcendental Foundation of Quantum Objectivity (Walter de Gruyter, Berlin, New York, 2007) 1-8.

30. Cf. M. Giovanelli, Reality and Negation-Kant's Principle of Anticipations of Perception. An Investigation of Its Impact on the Post-Kantian Debate, Studies in German Idealism, Vol. 11 (Springer, Dordrecht-Heidelberg-London- New York, 2011).

31. Cf. M. A. González Porta, op. cit. 
LO RELATIVO Y LO ABSOLUTO EN LA CONCEPCIÓN DE PAUL NATORP y Porta, evidenciando el valor de su teoría para los problemas de la filosofía de la ciencia contemporánea. 
